\title{
Árvore de classificação para identificação de Padrão respiratório ineficaz em crianças com infecção respiratória aguda
}

\author{
Daniel Bruno Resende Chaves ${ }^{1}$, Lívia Maia Pascoal2 ${ }^{2}$, Beatriz Amorim Beltrão ${ }^{3}$, Tânia Alteniza Leandro4, \\ Marília Mendes Nunes ${ }^{5}$, Viviane Martins da Silva ${ }^{6}$, Marcos Venícios de Oliveira Lopes ${ }^{7}$
}

\section{RESUMO}

O estudo teve como objetivo verificar as características definidoras com melhor poder de predição para auxiliar na classificação de Padrão respiratório ineficaz, utilizando árvores de classificação, em crianças com infeç̧ão respiratória aguda. Estudo transversal, realizado em dois hospitais pediátricos juntamente a 249 crianças com infecção respiratória aguda. Para a coleta, foi utilizado um instrumento específico desenvolvido para o estudo. Empregaram-se três algoritmos de indução para a geração das árvores, CHi-square Automatic Interaction Detection, Classification and Regression Trees e Quick, Unbiased, Efficient Statistical Tree. Construíram-se três árvores para auxiliar na identificação de Padrão Respiratório ineficaz. As árvores de classificação geradas apresentam probabilidades condicionais à ocorrência do diagnóstico associada a dispneia e alterações na profundidade respiratória. Padrão respiratório ineficaz esteve presente $65,5 \%$ da amostra. Assim, a probabilidade da ocorrência do referido diagnóstico nas crianças com infecção respiratória aguda foi de $100 \%$ com a presença de dispneia e de alterações na profundidade respiratória.

Descritores: Árvores de Decisões; Saúde da Criança; Enfermagem Pediátrica.

\footnotetext{
${ }^{1}$ Enfermeiro, Doutor em Enfermagem. Enfermeiro do Hospital Geral Dr. Waldemar Alcântara. Fortaleza, CE, Brasil. E-mail: dbresende@yahoo.com.br.

${ }^{2}$ Enfermeira, Doutora em Enfermeira. Professora Adjunta da Universidade Federal do Maranhão. Imperatriz, MA, Brasil. E-mail: livia_mp@hotmail.com.

${ }^{3}$ Enfermeira, Mestre em Enfermagem. Discente do Programa de Pós-Graduação em Enfermagem da Universidade Federal do Ceará, nível Doutorado. Enfermeira da Unidade de Terapia Intensiva do Hospital Universitário Walter Cantídio do Universidade Federal do Ceará. Fortaleza, CE, Brasil. E-mail: biaamorimm@yahoo.com.br.

${ }^{4}$ Enfermeira, Mestre em Enfermagem. Discente do Programa de Pós-Graduação em Enfermagem da Universidade Federal do Ceará, nível Doutorado. Enfermeira Assistencial do Instituto do Câncer do Ceará. Fortaleza, CE, Brasil. E-mail: taniallt@yahoo.com.br.

${ }^{5}$ Enfermeira, Mestre em Enfermagem. Discente do Programa de Pós-Graduação em Enfermagem da Universidade Federal do Ceará, nível Doutorado. Fortaleza, CE, Brasil. E-mail: marilia mn@hotmail.com.

${ }^{6}$ Enfermeira, Doutora em Enfermagem. Professora Associada da Universidade Federal do Maranhão. Imperatriz, MA, Brasil. E-mail: viviane.silva@outlook.com.

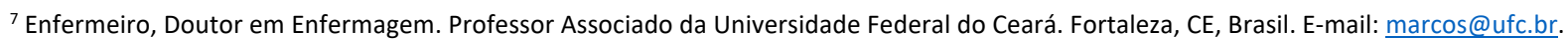

\section{Como citar esse artigo:}

Chaves DBR, Pascoal LM, Beltrão BA, Leandro TA, Nunes MM, Silva VM, et al. Árvore de classificação para identificação de Padrão respiratório ineficaz em crianças com infecção respiratória aguda. Rev. Eletr. Enf. [Internet]. 2018 [acesso em: ];20:v20a45. Disponível em: https://doi.org/10.5216/ree.v20.45401. 


\section{INTRODUÇÃO}

A tomada de decisão diagnóstica constitui um dos elementos essenciais para determinar uma assistência de qualidade e bons resultados clínicos. Entretanto, várias condições podem dificultar ou gerar confundimento em uma inferência diagnóstica precisa, tais como a falta de familiaridade dos enfermeiros com os diagnósticos de enfermagem, a subjetividade dos indicadores clínicos e da inferência diagnóstica, o uso de sinais e sintomas comuns e a sobreposição de diagnósticos ${ }^{(1)}$. Além disso, em muitas situações, o enfermeiro necessita decidir rapidamente se uma condição clínica representa o verdadeiro status do paciente, baseado em um pequeno número de indicadores clínicos, gerando um ambiente de incerteza.

As árvores de classificação (AC) são ferramentas que têm o objetivo de predizer um desfecho a partir de variáveis preditoras ${ }^{(2)}$. Estas árvores são elementos gráficos utilizados para calcular as probabilidades de um indivíduo apresentar um diagnóstico de enfermagem, considerando a presença/ausência de um pequeno conjunto de indicadores clínicos. Estes elementos gráficos permitem uma visão global de múltiplas combinações de status clínicos com uma medida de probabilidade para cada status. Isto permite uma decisão diagnóstica rápida e eficiente, facilitando o processo de raciocínio clínico, bem como permitindo decidir precocemente a conduta sobre a terapêutica a ser instituída, de modo a produzir bons resultados de enfermagem ${ }^{(3)}$.

Ao se utilizar árvores de classificação, a tomada de decisão diagnóstica é tratada como um processo de redução da incerteza baseado em probabilidades condicionadas, obtidas a partir de informação clínica disponível num dado momento ${ }^{(3)}$. Este processo é particularmente útil em situações que requerem uma triagem diagnóstica e que representam o comprometimento de uma função vital. Neste ponto, crianças com Infecção respiratória aguda (IRA) podem apresentar alterações do processo ventilatório que se gravem rapidamente e necessitar de suporte ventilatório. Assim, o desenvolvimento de uma árvore de classificação para a identificação rápida de um diagnóstico Padrão respiratório ineficaz (PRI) pode ser uma ferramenta útil para os enfermeiros.

Padrão respiratório ineficaz é um diagnóstico de enfermagem, incluído na NANDA-I em 1980 e, atualmente, é definido como "inspiração e/ou expiração que não proporciona uma ventilação adequada". Este diagnóstico tem como características definidoras: alterações na profundidade respiratória; assumir uma posição de três pontos; batimentos de asa do nariz; bradipneia; capacidade vital diminuída; diâmetro ântero-posterior aumentado; dispneia; excursão torácica alterada; fase de expiração prolongada; ortopneia; pressão expiratória diminuída; pressão inspiratória diminuída; respiração com os lábios franzidos; taquipneia; uso da musculatura acessória para respirar; e ventilação-minuto diminuída( ${ }^{(4)}$.

As infecções em vias respiratórias são problemas comuns em crianças e constituem o principal motivo de procura ao serviço hospitalar ${ }^{(5-6)}$, representando mundialmente uma das principais causas de morbidade e mortalidade em crianças menores de cinco anos ${ }^{(7)}$.

Na temática de diagnósticos de enfermagem respiratórios na população infantil, observou-se que muitos dos estudos realizados anteriormente possuíam como objetivo apresentar o perfil dos diagnósticos. Estudos desenvolvidos com crianças com IRA e PRI foram frequentemente encontrados ${ }^{(8-9)}$. Embora estas pesquisas tenham clarificado muitas questões, o processo de inferência e tomada de decisão na prática clínica ainda não havia sido contemplado. 
Com base no exposto, o objetivo da pesquisa foi identificar as características definidoras com melhor poder de predição, utilizando Árvores de Classificação, para auxiliar na classificação correta de Padrão respiratório ineficaz, em crianças com infecção respiratória aguda.

\section{METODOLOGIA}

Estudo do tipo transversal, descritivo e de natureza quantitativa desenvolvido com 249 crianças com comprometimento respiratório, internadas em dois hospitais pediátricos na região Nordeste do Brasil. A amostra foi selecionada de forma consecutiva. Para participar do estudo, a população devia ter diagnóstico médico de Infecção respiratória aguda e ter no máximo cinco anos de idade completos. O tamanho amostral foi determinado considerando o número de características definidoras. O estudo adotou uma estimativa de 15 crianças para cada característica definidora (total de 16). Portanto, o tamanho amostral foi estimado em 240 crianças com IRA.

A coleta dos dados foi realizada por seis enfermeiros previamente treinados em uma oficina de oito horas. Esta foi desenvolvida com o intuito de padronizar o procedimento de coleta de dados, composta com os seguintes tópicos: métodos propedêuticos inerentes à avaliação respiratória, semiologia geral e pediátrica e discussão sobre o diagnóstico PRI. O instrumento de coleta dos dados foi desenvolvido com base na literatura pertinente acerca da avaliação pulmonar e submetido à apreciação de dois professores com experiência em semiologia e diagnósticos de enfermagem.

O instrumento proporcionou o levantamento de variáveis relacionadas à identificação das crianças e à avaliação respiratória, incluindo as características definidoras do diagnóstico em estudo citadas anteriormente. Destaca-se que quatro características não puderam ser avaliadas nesta pesquisa devido à idade da população e à impossibilidade de se realizar testes de função pulmonar necessários para mensurar esses indicadores: capacidade vital diminuída, pressão expiratória diminuída, pressão inspiratória diminuída, e ventilação-minuto diminuída.

Foram organizadas planilhas com informações de cada criança sobre a presença ou a ausência das características definidoras de PRI e analisadas por enfermeiros diagnosticadores para determinar a presença ou ausência do diagnóstico citado. A ocorrência ou não do diagnóstico foi determinada pela concordância absoluta entre os diagnosticadores. Para participar desta etapa os enfermeiros deveriam atender aos seguintes critérios: publicação de pesquisas sobre diagnósticos, intervenções ou resultados de enfermagem e prática docente ou clínica, além disso, foram treinados e submetidos a testes para avaliação de atributos e qualificação das inferências realizadas.

Para a geração das árvores optou-se por trabalhar com três algoritmos de indução de Árvores de classificação: CHi-square Automatic Interaction Detection (CHAID), Classification and Regression Trees (CART) e Quick, Unbiased, Efficient Statistical Tree (QUEST). Para indução das árvores, o diagnóstico PRI foi adotado como variável dependente e as características definidoras, como variáveis independentes que iriam compor os nós secundários e ramos da árvore.

Para a geração da AC com o algoritmo CHAID, utilizaram-se: nível de significância para a divisão dos nós e fusão de categorias de 0,05, razão de verossimilhança como método para obter o valor Qui-Quadrado, número máximo de 100 interações e alteração mínima das frequências esperadas das caixas de 0,05 para estimação do 
modelo. Os valores de significância para os métodos, os parâmetros de divisão e fusão foram corrigidos pelo método de Bonferroni.

A AC gerada com o método de crescimento CART utilizou a medida GINI como parâmetro para reduzir as impurezas necessárias na divisão dos nós. Esta toma como base o quadrado das probabilidades de pertença dos casos em cada categoria da variável dependente. Estabeleceu-se 0,001 como valor para redução mínima das impurezas na divisão do nó. Para o algoritmo QUEST, estabeleceu-se nível de significância para a divisão dos nós de 0,05. O QUEST é um método rápido e evita vieses que outros métodos apresentam, por favorecer os preditores com muitas categorias.

Independentemente do método de geração a ser utilizado, foram determinados limites de crescimento. Estes variaram de acordo com o próprio método de geração, o nível de medida de uma variável dependente ou a combinação entre ambos. Adotou-se o número máximo de três níveis para o algoritmo CHAID e de cinco níveis para CART e QUEST. Determinou-se número mínimo de casos de 50 para o nó raiz e de 20 para os nós derivados. Estes parâmetros foram utilizados com o intuito de gerar todas as relações possíveis entre as variáveis.

O critério da poda da árvore foi utilizado para evitar um superajustamento do modelo com o emprego dos algoritmos CART e QUEST. Assim, a árvore foi recortada de modo a se obter uma subárvore menor baseada na especificação da máxima diferença do risco, o qual é expresso em erros típicos. O valor do risco determinado no presente estudo foi de zero.

Neste estudo uma avaliação da qualidade da estrutura das árvores geradas foi realizada por meio da validação cruzada. Esta divide a amostra em um número pré-estabelecido de subamostras (máximo de 25 subamostras). Por conseguinte, são geradas várias árvores, sempre se excetuando um subgrupo de dados gerais. Para cada árvore, calcula-se o risco de erro de classificação, aplicando-se a árvore à subamostra que foi excluída antes de esta ser gerada. Como resultado final, tem-se uma única árvore cuja estimativa do risco é calculada pela média dos riscos de todas as árvores. Então, as árvores geradas na pesquisa podem ser utilizadas de forma generalizada.

Para realizar estas análises processaram-se os dados no software IBM SPSS versão 19.0 for Windows, adotando nível de significância de 0,05.

O estudo obedeceu aos aspectos éticos sobre pesquisa com seres humanos, sendo aprovado pelo Comitê de Ética em Pesquisa com número de protocolo 309/10. Buscou-se a anuência dos pais mediante a assinatura do Termo de Consentimento Livre e Esclarecido. Este também foi assinado pelos enfermeiros diagnosticadores que participaram da pesquisa.

\section{RESULTADOS}

O diagnóstico de enfermagem PRI foi identificado em 65,5\% das 249 crianças com IRA.

Construíram-se três AC para o diagnóstico de enfermagem PRI. Estas apresentam probabilidades condicionais à ocorrência associada às características definidoras do diagnóstico. Desta forma, pôde-se estimar a probabilidade de predição de cada conjunto de dados para PRI.

O método de crescimento CHAID foi utilizado para a geração da primeira árvore. Esta apresenta sete nós, sendo quatro nós terminais. As relações entre as variáveis foram expressas em dois níveis de profundidade e duas 
características foram consideradas relevantes para a construção desta árvore: dispneia e alterações na profundidade respiratória (Figura 1).

Figura 1: Árvore de classificação gerada com as características definidoras do diagnóstico de enfermagem Padrão respiratório ineficaz, utilizando-se o método de crescimento CHAID.

\begin{tabular}{|lcc|}
\hline \multicolumn{3}{|c|}{ Nó $\mathbf{0}$} \\
Categoria & $\%$ & $\mathrm{n}$ \\
\hline Sem DE & 34,5 & 86 \\
\hline Com DE & 65,5 & 163 \\
\hline \hline Total & 100,0 & 249 \\
\hline
\end{tabular}
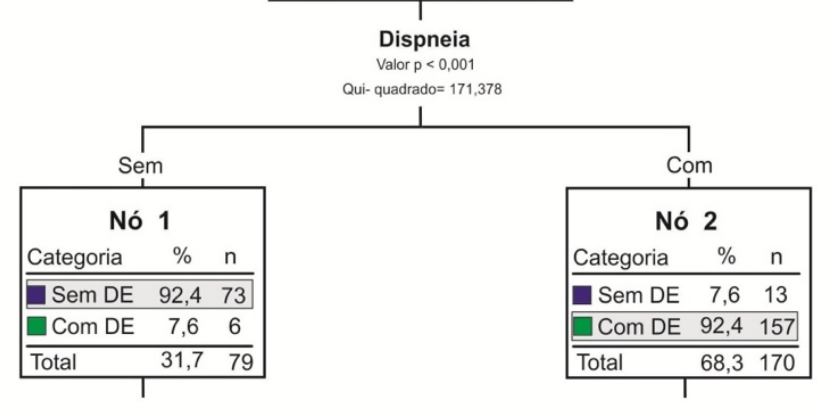

Alterações na profundidade respiratória Valor $p=0,012$ Qui- quadrado $=6,331$
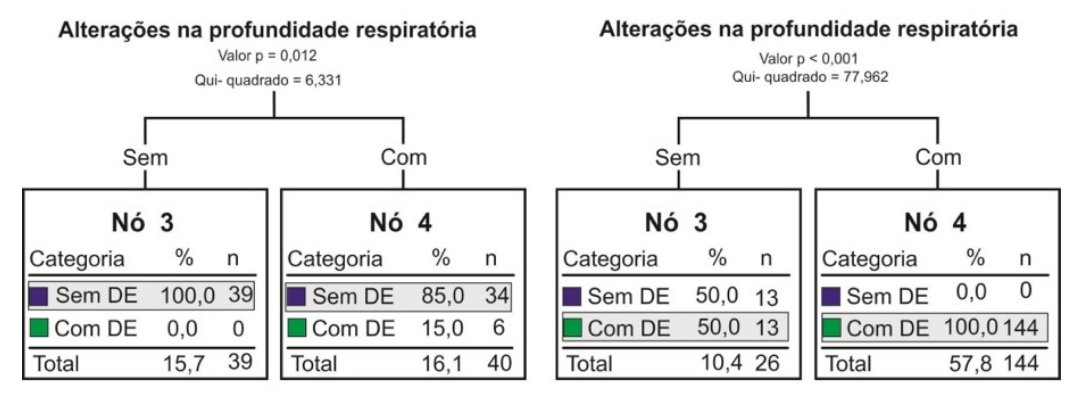

A característica definidora mais fortemente associada ao PRI foi dispneia. As crianças que manifestaram essa característica tiveram a probabilidade de ocorrência de PRI de 92,4\%. Para crianças que manifestaram dispneia e alterações na profundidade respiratória em conjunto, a probabilidade de ocorrência de PRI foi de $100 \%$.

As crianças sem a característica dispneia, dados do lado esquerdo da árvore referente, apresentaram baixa probabilidade de manifestação de PRI (7,6\%). Quando a dispneia estava ausente, mas as crianças manifestavam alterações na profundidade respiratória, a probabilidade de desenvolver PRI foi de 15\%. A ausência das duas características definidoras citadas apresentou probabilidade nula de ocorrência de PRI. Esta AC gerada pelo algoritmo CHAID e mostrou um poder de predição de 92,4\%.

Na segunda árvore gerada para o PRI, foi aplicado o método de crescimento CART. Esta possui cinco nós, sendo três terminais. De maneira similar à árvore gerada pelo método CHAID, a árvore apresenta as relações entre as características em dois níveis de profundidade, utilizando como mais relevantes para PRI as características dispneia e alterações na profundidade respiratória (Figura 2). A predição da árvore pelo método de crescimento CART também foi de 92,4\%. Dispneia foi a característica mais fortemente associada, com a probabilidade de ocorrência do diagnóstico PRI de 92,4\%. A presença de dispneia e alterações na profundidade respiratória também apresentou valor máximo para manifestação de PRI (100\%). Observando-se o lado esquerdo da árvore, percebeuse que as crianças que não possuíam a característica dispneia tinham baixa probabilidade de apresentar PRI (7,6\%).

A AC gerada com o método de crescimento QUEST apresenta similaridade com as outras duas induzidas para PRI. A árvore possui cinco nós no total, sendo três terminais, bem como apresenta dois níveis de 
profundidade. As características definidoras utilizadas para a construção da árvore são as mesmas empregadas nas árvores anteriores: dispneia e alterações na profundidade respiratória (Figura 3).

Figura 2: Árvore de classificação gerada com as características definidoras do diagnóstico de enfermagem Padrão respiratório ineficaz, utilizando-se o método de crescimento CART.

\begin{tabular}{|lcc|}
\hline \multicolumn{3}{|c|}{ Nó $\mathbf{0}$} \\
Categoria & $\%$ & $\mathrm{n}$ \\
\hline Sem DE & 34,5 & 86 \\
\hline Com DE & 65,5 & 163 \\
\hline Total & 100,0 & 249 \\
\hline \multicolumn{2}{|c}{ Dispneia } \\
\hline
\end{tabular}

Melhoramento $=0,311$
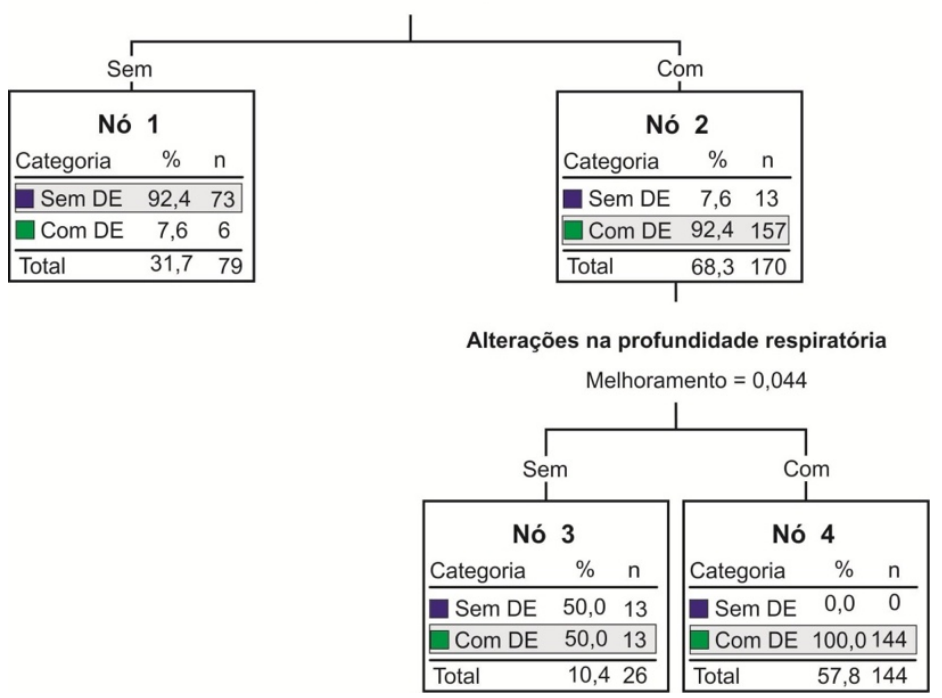

Figura 3: Árvore de classificação gerada com as características definidoras do diagnóstico de enfermagem Padrão respiratório ineficaz, utilizando-se o método de crescimento QUEST.

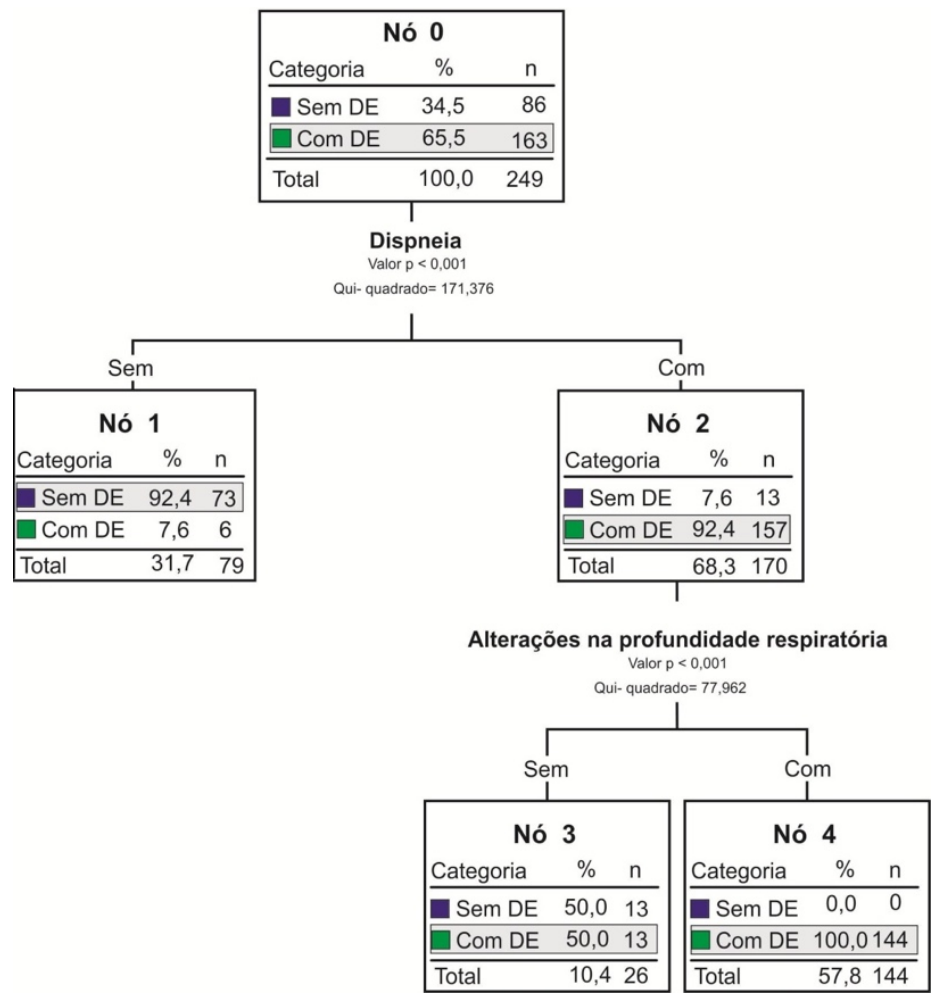


Os valores identificados foram os mesmos, a dispneia foi mais fortemente associada, com $92,4 \%$ de probabilidade de ocorrência do diagnóstico diante desta característica. A regra dedutível presença de dispneia e alterações na profundidade respiratória determinou probabilidade de 100\% para a ocorrência de PRI. O poder de predição global da árvore pelo método QUEST, calculado pelo método de validação cruzada, foi de $92,4 \%$.

\section{DISCUSSÃO}

Crianças que desenvolvem PRI apresentam inspiração e/ou expiração que não proporcionam ventilação

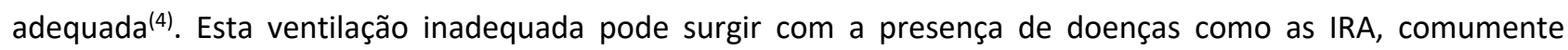
observadas na população infantil(10). Portanto, dado o comprometimento respiratório, é importante que o enfermeiro possa conhecer melhor o diagnóstico citado e saber como ele se comporta em uma população específica.

A maior parte das crianças avaliadas, neste estudo, apresentou Padrão respiratório ineficaz. Este diagnóstico também é identificado em outras pesquisas com crianças em situações clínicas diversas ${ }^{(11-13)}$. Nesse contexto, percebe-se que a manifestação e prevalência elevada de PRI podem surgir em decorrência das manifestações clínicas decorrentes de infecções respiratórias apresentadas pela população específica. Esta afirmativa é corroborada em pesquisas realizadas anteriormente sobre diagnósticos de enfermagem respiratórios em crianças com IRA ${ }^{(9,14)}$.

As três AC geradas para auxiliar na inferência de PRI também mantiveram uma estrutura padronizada, elas apresentaram dispneia e alterações na profundidade respiratória na sua estrutura. Em todas as árvores geradas, a probabilidade da ocorrência do diagnóstico de enfermagem PRI foi de $100 \%$ com a presença de dispneia e de alterações na profundidade respiratória. As três árvores apresentaram o mesmo poder de predição global. Estas predisseram corretamente PRI em $92,4 \%$ dos casos. A AC obtida pelo método de crescimento CHAID, apresentou uma ramificação adicional em comparação às outras árvores. A regra expressa pela ausência das características dispneia e alterações na profundidade respiratória apresentou probabilidade nula de ocorrência de PRI.

Nesse sentido, as características definidoras apresentadas anteriormente são representativas de PRI. De acordo com a análise multivariada dos dados, as árvores de classificação geradas apresentam probabilidades condicionais à ocorrência de PRI associada a dispneia e alterações na profundidade respiratória. Estas correspondem a um processo de compensação do corpo humano para auxiliar o padrão respiratório ${ }^{(15)}$. Já a dispneia refere-se a uma condição na qual há um desconforto ou dificuldade para respirar, surgindo como consequência de complicações ou falha nos mecanismos de defesa(15-16).

Alterações na profundidade respiratória foi o indicador clínico mais prevalente na população da pesquisa, sendo manifestado por alterações no ritmo respiratório, frequência respiratória e/ou utilização de músculos acessórios. Ao avaliar a presença de diagnósticos de enfermagem em crianças com sinais e sintomas respiratórios, alterações na profundidade respiratória apresentou associação estatística significante com PRI e esteve presente em $73,3 \%$ da população ${ }^{(8)}$. De maneira similar, outros pesquisadores ${ }^{(9)}$ verificaram que o referido indicador apresentou bons valores de sensibilidade, apontando que na presença deste o diagnóstico muito provavelmente estará presente. Destaca-se que o indicador alterações na profundidade respiratória pode ser influenciado por 
outros sinais de PRI. Como exemplo, o indicador dispneia, quando manifestado, vem associado a alterações na profundidade respiratória.

Dispneia foi a segunda característica definidora de PRI mais prevalente na população estudada, por ser um indicador associado ao esforço respiratório(10), a respiração fica difícil, desconfortável e cansativa ${ }^{(17)}$. Situação esta apresentada por 170 (68,3\%) participantes. De forma similar, em outras pesquisas, ao se avaliar os diagnósticos de enfermagem respiratórios em crianças, a dispneia esteve presente em 75\% (102) e 99\% (203) da população(1819).

Ao investigar esse indicador, observa-se que a dispneia está fortemente associada à presença de PRI, dados esses corroborados por estudos anteriores, em que o indicador clínico apresentou associação estatística significante com o diagnóstico ${ }^{(17)}$ e esteve associado a um aumento em 15 ou 36 vezes da chance de apresentar o $P R I^{(14,20)}$. Além disso, dispneia apresentou valores de sensibilidade significantes para o diagnóstico ${ }^{(9,20-21)}$.

Dessa forma, a identificação correta do conjunto de indicadores que representam um desfecho é crucial para que o enfermeiro possa prestar uma assistência adequada e de qualidade. Porém, na prática clínica nem sempre é fácil para o enfermeiro identificar esse conjunto de respostas humanas, dadas as peculiaridades de cada paciente. Portanto, a utilização de ferramentas tecnológicas, como as AC, auxilia no processo de inferência diagnóstica acurada.

\section{CONCLUSÃO}

As crianças avaliadas com IRA apresentaram alta prevalência de Padrão respiratório ineficaz. As três árvores desenvolvidas com base nos métodos CHAID, CART e QUEST auxiliaram na inferência do diagnóstico de PRI. Assim, pode-se identificar a ocorrência deste diagnóstico em $100 \%$ dos casos, quando dispneia e alterações na profundidade respiratória estavam presentes.

Destaca-se a escassez de estudos com desenhos metodológicos semelhantes a esta pesquisa, principalmente na área da enfermagem, o que limitou a comparação dos resultados. No entanto, ressaltamos que as árvores geradas são ferramentas tecnológicas inovadoras e eficazes para calcular as probabilidades de uma criança apresentar PRI por meio de um conjunto de indicadores. Essa metodologia proporciona ao enfermeiro uma visão ampla sobre as variações clínicas das crianças e estimula o processo de raciocínio diagnóstico. Portanto, sugere-se que novos estudos sejam realizados no intuito de aplicar as árvores geradas na prática clínica na população específica e em outros grupos populacionais.

\section{REFERÊNCIAS}

1. Leandro TA, Araújo TL, Cavalcante TF, Lopes MVO, Oliveira TMF, Lopes ACM. Urinary incontinence nursing diagnoses in patients with stroke. Revista da Escola de Enfermagem da USP [Internet]. 2015 [acesso em: 4 abr. 2018];49(6):923-30. Disponível em: http://dx.doi.org/10.1590/S0080-623420150000600007.

2. Lemus IS, Zamora CS. Árboles de clasificación y regresión: modelos Cart. Ciencia \& Trabajo. 2008;10(30):161-6.

3. Lopes MVO, Silva VM. Métodos avançados de validação de diagnósticos de enfermagem. In: Herdman TH, Napoleão AA, Lopes CT, Silva VM (Org.). PRONANDA: Programa de atualização em diagnósticos de enfermagem. 3a ed. Porto Alegre: Artmed; 2016. p.31-74.

4. NANDA International. Diagnósticos de enfermagem da NANDA: definições e classificação 2009-2011. Porto Alegre: Artmed; 2010. 
5. Pfuntner A, Wier LM, Stocks C. Most frequent conditions in U.S. hospitals, 2011. HCUP Statistical Brief\#162. Agency for Healthcare Research and Quality [Internet]. 2013 [acesso em: 28 fev. 2018]. Disponível em: http://www.hcup-

us.ahrq.gov/reports/statbriefs/sb162.pdf

6. Oliveira TG, Moraes JSB, Moreira FT, Arrelaro RC, Ricardi VA, Bertagnon JRD, et al. Evaluation of hospitalization of children aged 0 to 5 years admitted for respiratory infections at a large hospital. Einstein [Internet]. 2011 [acesso em: 27 fev. 2018];9(4):514-7. Disponível em: http://dx.doi.org/10.1590/s1679-45082011ao1908

7. Theodoratou E, McAllister DA, Reed C, Adeloye DO, Rudan I, Muhe LM, et al. Global, regional, and national estimates of pneumonia burden in HIV-infected children in 2010: a meta-analysis and modelling study. Lancet Infect Dis [Internet]. 2014 [acesso em: 24 fev. 2018];14(12):1250-8. Disponível em: http://dx.doi.org/10.1016/\$1473-3099(14)70990-9.

8. Chagas KLM, Lima LHO, Oliveira EAR, Luz GOA. Diagnósticos de enfermagem em crianças com sinais e sintomas respiratórios: um estudo descritivo. Rev Rene [Internet]. 2011 [acesso em: 12 fev. 2017];12(2):302-8. Disponível em:

http://www.revistarene.ufc.br/revista/index.php/revista/article/viewFile/157/68

9. Pascoal LM, Lopes MVO, Silva VM, Beltrão BA, Chaves DBR, Santiago JMV, et al. Ineffective Breathing Pattern: Defining Characteristics in Children With Acute Respiratory Infection. Int J Nurs Terminol Knowledge [Internet]. 2014 [acesso em: 24 fev. 2018];25(1):54-61. Disponível em: http://dx.doi.org/10.1111/j.2047-3095.2013.01249.x.

10. Hockenberry MJ, Wilson D. Wong: fundamentos de enfermagem pediátrica. 9 ed. Rio de Janeiro: Elsevier; 2014.

11. Neves RS, Araujo PHM, Lacerda TCL. Diagnósticos de enfermagem prevalentes no bloco materno-infantil de um hospital público de Brasília. Enferm. Foco [Internet]. 2014 [acesso em: 25 fev. 2018];5(3/4):53-6. Disponível em:

http://revista.cofen.gov.br/index.php/enfermagem/article/view/560.

12. Avena MJ, Pedreira LGM, Herdman TH, Gutiérrez MGR. Respiratory Nursing Diagnoses: Presenting Evidence for Identification of the Defining Characteristics in Neonatal and Pediatric Populations. Int J Nurs Terminol Knowledge [Internet]. 2016 [acesso em: 26 fev. 2018];27:184-92. Disponível em: https://doi:10.1111/2047-3095.12098.

13. Nóbrega RV, Nóbrega MML, Silva KL. Diagnósticos, resultados e intervenções de enfermagem para crianças na Clínica Pediátrica de um hospital escola. Rev. Bras. Enferm [Internet]. 2011 [acesso em: 8 fev. 2017];64(3):501-10. Disponível em:

http://dx.doi.org/10.1590/S0034-71672011000300014.

14. Andrade LZC, Chaves DBR, Silva VM, Beltrão BA, Lopes MVO. Respiratory nursing diagnoses for children with acute respiratory infection. Acta Paul Enferm [Internet]. 2012 [acesso em: 10 fev. 2017];25(5):713-20. Disponível em:

http://dx.doi.org/10.1590/S0103-21002012000500011.

15. West JB. Fisiopatologia Pulmonar: princípios básicos. 7 ed. Porto Alegre: Artmed; 2010.

16. Parshall MB, Schwartzstein RM, Adams L, Banzett RB, Manning HL, Bourbeau J, et al. An official American thoracic society statement: update on the mechanisms, assessment, and management of dyspnea. Am J Respir Crit Care Med [Internet]. 2012 [acesso em: 25 fev. 2018];185(4):435-52. Disponível em: http://dx.doi.org/10.1164/rccm.201111-2042ST.

17. Cavalcante JCB, Mendes LC, Lopes MVO, Lima LHO. Indicadores clínicos de padrão respiratório ineficaz em crianças com asma. Rev Rene [Internet]. 2010 [acesso em: 9 fev. 2017];11(1):66-75. Disponível em:

http://www.periodicos.ufc.br/rene/article/view/4476.

18. Pascoal LM, Lopes MVO, Silva VM, Beltrão BA, Chaves DBR, Nunes MM, et al. Prognostic clinical indicators of short-term survival for ineffective breathing pattern in children with acute respiratory infection. Journal of Clinical Nursing [Internet]. 2015 [acesso em: 28 fev. 2018];25:752-9. Disponível em: http://dx.doi.org/10.1111/jocn.13064.

19. Carvalho OMC, Silva VM, Távora RCO, Chaves DBR, Beltrão BA, Lopes MVO. Adecuación de las características definitorias en los diagnósticos de deterioro del intercambio de gases y de la ventilación espontánea en ninos asmáticos. Enferm Clin [Internet]. 2015 [acesso em: 27 fev. 2018];25(6):296-304. Disponível em: http://dx.doi.org/10.1016/j.enfcli.2015.07.007.

20. Silveira UA, Lima LHO, Lopes MVO. Características definidoras dos diagnósticos de enfermagem Desobstrução ineficaz das vias aéreas e Padrão respiratório ineficaz em crianças asmáticas. Rev Rene [Internet]. 2008 [acesso em: 9 fev. 2017];9(4):125-

33. Disponível em: http://www.periodicos.ufc.br/rene/article/view/5248.

21. Beltrão BA, Herdman TH, Pascoal LM, Chaves DB, da Silva VM, et al. Ineffective breathing pattern in children and adolescents with congenital heart disease: accuracy of defining characteristics. J Clin Nurs [Internet]. 2015 [acesso em: 3 abr. 2018];24(17-

18):2505-13. Disponível em: http://dx.doi.org/10.1111/jocn.12838. 